\title{
Building the RHIC Tracking Lattice Model
}

\author{
Y. Luo, W. Fischer, S. Tepikian
}

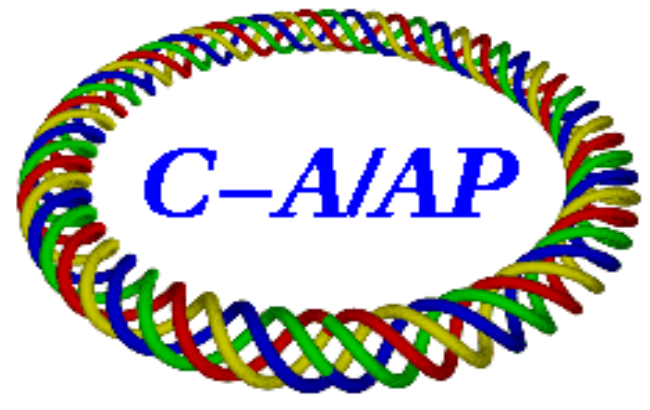

\section{Collider-Accelerator Department Brookhaven National Laboratory \\ Upton, NY 11973}

Notice: This document has been authorized by employees of Brookhaven Science Associates, LLC under Contract No. DE-AC02-98CH10886 with the U.S. Department of Energy. The United States Government retains a nonexclusive, paid-up, irrevocable, world-wide license to publish or reproduce the published form of this document, or allow others to do so, for United States Government purposes. 
January 26, 2010

\title{
Building the RHIC tracking lattice model
}

\author{
Y. Luo, W. Fischer and S. Tepikian \\ Brookhaven National Laboratory, Upton, NY 11973, USA
}

In this note we outline the procedure to build a realistic lattice model for the RHIC beam-beam tracking simulation. We will install multipole field errors in the arc main dipoles, arc main quadrupols and interaction region magnets ( DX, D0, and triplets ) and introduce a residual closed orbit, tune ripples, and physical apertures in the tracking lattice model. Nonlinearities such as local IR multipoles, second order chromaticies and third order resonance driving terms are also corrected before tracking.

\section{Introduction}

In the calculation of dynamic aperture and beam-beam lifetime, a realistic lattice model is required to benchmark the current RHIC observation and to predict the future operation. The tracking lattice model [1] we are currently using is incomplete. For example, the multipole field errors in the arc main dipoles and quadrupoles, the effect of closed orbit are ignored. Up to now in our dynamic aperture calculation the beam-beam interaction is modeled as a 4-D transverse kick, which is not justified by the fact that the proton bunch length is comparable to the $\beta^{*}$ at the collisonal interaction points (IPs). The goal of this note is to include all the known errors and noise sources into the tracking lattice model.

The tracking code we are using most is SixTrack [2]. SixTrack is a fast symplectic tracking code. It is an executable. Therefore it lacks flexibilities to introduce a new type of element or to adjust the parameters of beam during tracking. To overcome that, Y. Luo developed a $\mathrm{C}++$ accelerator tracking library called SimTrack which supplies interfaces to modify beam line and element parameters and to introduce a new type of element. SimTrack uses a 4-th order symplectic integerator [3] for optical tracking. Considering SixTrack's speed and SimTrack's flexibility, we will use both of them in our future tracking simulation. Therefore our tracking lattice model will be presented in the input files of SixTrack or SimTrack.

In the following, starting from the base lattice model in the format of MADX [4], we first install multipole filed errors to the interaction region (IR) magnets, arc main dipoles and main quadruples in the tracking lattice model, followed by the nonlinear corrections. Then we include closed orbit deviations, tune ripples, physical aperture and beam-beam integration.

\section{Base lattice model}

The base lattice model of the RHIC rings defines the elements and line sequences, together with the initial strengths for the main dipoles and quadrupoles for a certain RHIC run. The base lattice model of the RHIC is normally given in the input files of MAD8 and MADX [5]. For example, following is a MADX input file for the 2009 RHIC $250 \mathrm{GeV}$ polarized proton (pp) run.

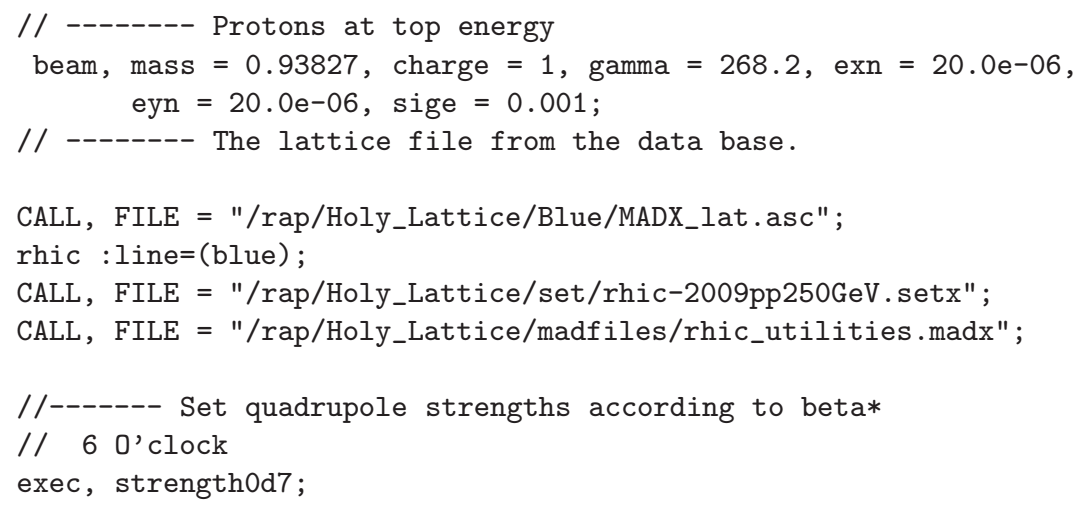




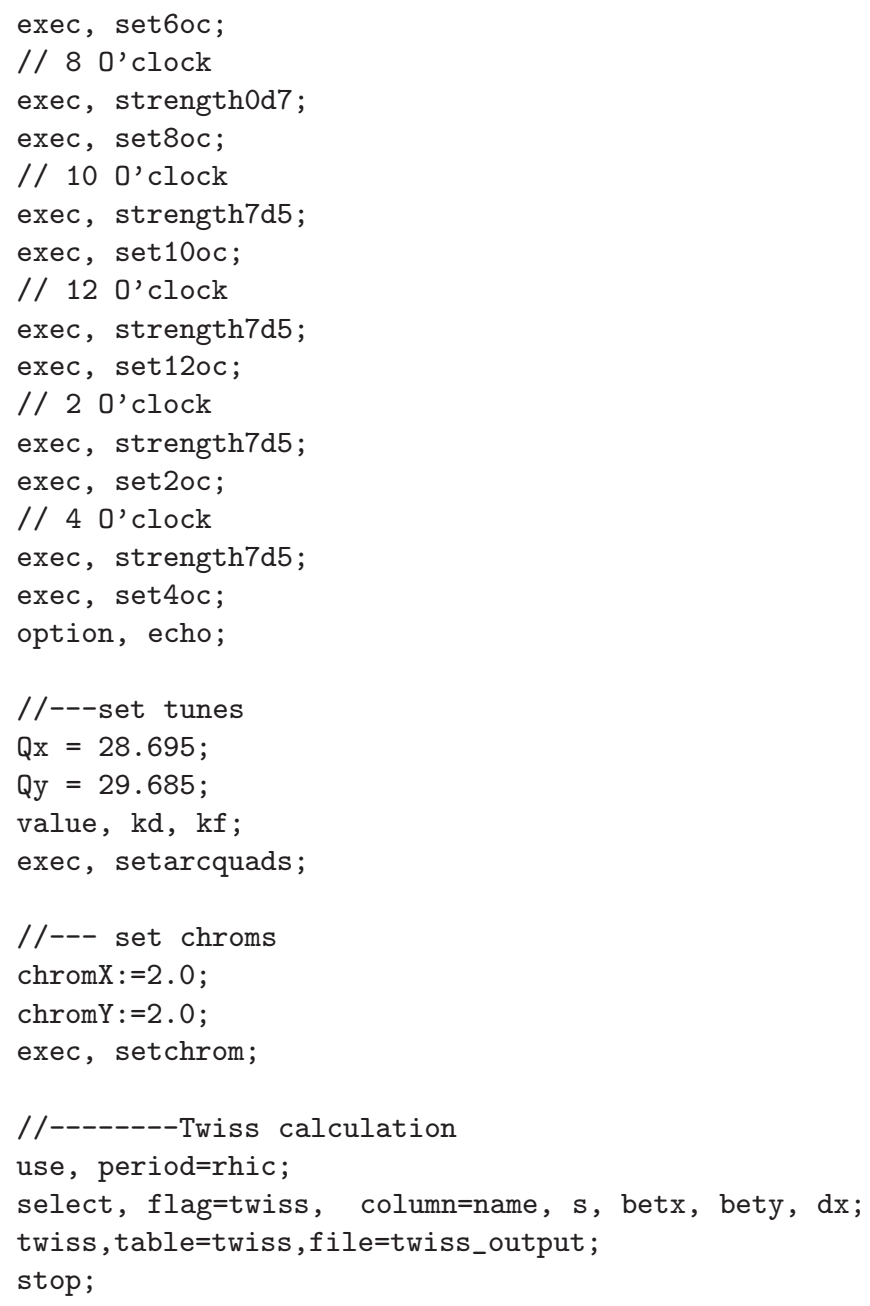

This file calls three input files: MADX_lat.asc, rhic_2009pp250GeV.setx, rhic_utilities.madx. MADX_lat.asc defines elements and sequences. rhic_2009pp250GeV.setx gives the initial strengths for the main dipoles and quadrupoles for RHIC $2009250 \mathrm{GeV}$ pp run. rhic_utilities.madx supplies some MADX macros to fit tunes and chromaticities. For each RHIC run, there will be a specific *. setx file ( setx means strength settings in MADX format ). All of the files are kept under the directory "/rap/Holy_Lattice". The second part of this file is to set the values of beta functions at the interaction points (IPs). The last part is to set the tunes and linear chromaticities.

\section{3 "parameters_input" and converters}

To facilitate the exchange of lattice data between accelerator design codes and to simplify the procedure to modify the elements in the MADX input file, we create a generic format to hold all magnetic parameters. To generate this file in MADX, we simply include the following block.

//----generic ring parameter output

use, period=rhic;

select, flag=twiss, column=NAME, KEYWORD, S, L, ANGLE, E1, E2, tilt, KOL , KOSL, K1L, K1SL, K2L , K2SL , K3L , K3SL , K4L , K4SL , K5L , K5SL , K6L , K6SL , K7L , K7SL, K8L , K8SL , K9L , K9SL, K10L , K10SL, KS ; twiss, table=twiss, file=parameters_input;

stop;

The output file "parameters_input" includes each element's name, type, length, location and all magnetic strengths. The elements are listed one by one in the sequential order from the beginning to the end of the ring.

We already wrote several converters to read in the file "parameters_input" to generate the input files for MAD8, TRACY-II, SAD and even MADX. For example, we can easily delete, split and insert elements in 
"parameters_input". After that we use our converter "madxTomadx.py" to generate a new MADX input file which includes the modifications.

MADX generates the input files ( fort.2, fort.3, fort.16, etc.) for the SixTrack. The command is

SIXTRACK, RADIUS=0.04;

We avoid additional manipulatiions except tracking in SixTrack since SixTrack is not an interactive programming language. The file "parameters_input" can be read by SimTrack without any modification. SimTrack supplies the interfaces to add, to delete, to insert elements. Since it is a library in $\mathrm{C}++$, it is programmable.

\section{RHIC magnet field measurement convention}

Before we install the measured multipole errors for the RHIC magnets into the tracking lattice model, we first connect the MAD defined magnet strengths to the magnet multipole measurement in the RHIC.

In the convention of RHIC magnet field measurement $[6,7]$, the integrated magnetic field is

$$
\left(B_{y} L\right)+i\left(B_{x} L\right)=\left[B\left(R_{\text {ref }}\right) L\right]\left[10^{-4} \sum_{n=0}^{N_{\max }}\left(b_{n}+i a_{n}\right) \frac{(x+i y)^{n}}{R_{r e f}^{n}}\right],
$$

where $b_{n}$ and $a_{n}, n=0,1,2, \ldots$, are the measured normal and skew multipole field coefficients. $n=0$ represents the dipole field. $B\left(R_{\text {ref }}\right)$ is the main field at radius $R_{r e f} . \quad L$ is the magnet's length. $10^{-4}$ in Eq. (8) is used for normalization, for example, for the filed measurement of a dipole magnet, $b_{0}=10^{4}$.

In the MADX, we use the magnet strengths which are defined as

$$
\begin{aligned}
K_{n} & =\frac{1}{(B \rho)} \frac{\partial B_{y}^{(n)}}{\partial x^{n}}, \\
K_{n} S & =\frac{1}{(B \rho)} \frac{\partial B_{x}^{(n)}}{\partial x^{n}},
\end{aligned}
$$

where $n=0,1, \ldots$.

Connecting Eqs.(1-2) to Eqs.(3-4), for a dipole magnets, we obtain its multipole strengths in MADX convention,

$$
\begin{aligned}
K_{n} L & =\Delta \theta \times\left[10^{-4} \times b_{n}^{M} \times \frac{n !}{R_{r e f}^{n}}\right], \\
K_{n} S L & =\Delta \theta \times\left[10^{-4} \times a_{n}^{M} \times \frac{n !}{R_{r e f}^{n}}\right]
\end{aligned}
$$

where $\Delta \theta$ is the bending angle of the dipole magnet. For a quadrupole magnet, we have

$$
\begin{aligned}
K_{n} L & =K 1 L \times R_{\text {ref }} \times\left[10^{-4} \times b_{n}^{M} \times \frac{n !}{R_{r e f}^{n}}\right], \\
K_{n} S L & =K 1 L \times R_{\text {ref }} \times\left[10^{-4} \times a_{n}^{M} \times \frac{n !}{R_{r e f}^{n}}\right],
\end{aligned}
$$

where $K_{1} L$ is integrated strength of the quadrople magnet.

\section{Multipoloe errors in the IR magnets}

Magnetic multipole field errors in the RHIC IRs play a significant role in the reduction of the beam dynamic aperture at the store energy. These IR nonlinearities come from the magnetic imperfections in the triplets Q1, Q2 and Q3, and in the separation dipoles DX and D0. All of these magnets are superconducting.

Due to a tight installation schedule, only 13/24 Q3 magnets, 10/24 D0 magnets, 7/12 DX magnets, and all Q1 and Q2 magnets were measured cold. Even where cold measurements are available, the geometric configuration of the leads was not the same as the final as-installed magnet assemblies. To build a nonlinear IR model, magnet power supply currents were scaled from measuring one to the real operating one. If the magnet was not measured under cold conditions, the warm to cold conversions were used.

Efforts had been made in the past several years to create the IR nonlinear model [8, 9]. In the summer of 2008, the IR nonlinear models for both RHIC rings at both $100 \mathrm{GeV}$ and $250 \mathrm{GeV}$ are available. These IR multipole errors are presented in the format of MADX. They are kept under the directory "/rap/lattices/RHIC_nonlinear/". To include them in the MADX input, we simply include the following line, 


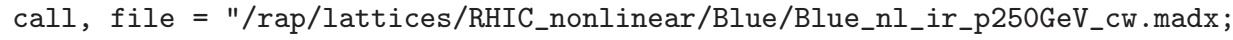

into the MADX input file. In this example, we included the IR multipole errors for the 2009 RHIC $250 \mathrm{GeV}$ pp run lattice.

\section{Multipole errors in the main dipoles and quadrupoles}

The arc main dipole and quadrupoles are also superconducting. Their multipole components were measured and reported in Ref. [10]. Since not all of the magnets were measured, we will randomly generate multipoles to the arc dipoles and quadrupoles according to the mean and rms values from the measured ones.

In the tracking lattice model we would like to include $b_{2}^{M}, b_{6}^{M}$ and $a_{2}^{M}$ into the bodies of arc main dipoles and include $b_{5}^{M}, b_{9}^{M}, a_{2}^{M}$ and $a_{5}^{M}$ into the bodies of arc main quadruoples. Table 1 lists the statistics of the measurements, which is excerpted from Table 7 and Table 9 in Ref. [10].

For simplicity, we split the main arc dipoles or quadrupoles into halves and insert a zero-length multipole between. The integrated strengths for these multipole slices are randomly assigned according Table 1 and Eqs. (3-4). This procedure is done with our scripts "split_D.py" and "split_Q.py" which modify the file "parameters_input". To generate a MADX input file with these modification, we just simply run our converter "madxTomadx.py".

Table 1: Measured multipoles components to be included in the tracking lattice model for $250 \mathrm{GeV}$

\begin{tabular}{lcc}
\hline \hline Order & Mean & RMS \\
\hline main arc dipole: & & \\
$b_{2}^{M}$ & +0.83 & +1.76 \\
$b_{6}^{M}$ & +1.19 & +0.14 \\
$b_{2}^{M}$ & -1.07 & +0.18 \\
main arc quadrupole: & & \\
$b_{5}^{M}$ & +5.70 & +0.62 \\
$b_{9}^{M}$ & -1.44 & +0.08 \\
$a_{2}^{M}$ & -1.83 & +1.68 \\
$a_{5}^{M}$ & -3.84 & +0.31 \\
\hline \hline
\end{tabular}

\section{Local nonlinear correction in the IRs}

In the manufacture of the RHIC triplet qudrupoles some multipole correcting spool pieces were integrated for the local nonlinear correction. In the RHIC operation, we use the method "IR bump" [11] to find these correctors' strengths.

In the tracking lattice model, knowing the multipole errors in the IRs, we use the analytical method "action-minimization" [12] to set these correctors' strengths. We first use MADX to generate a file named "test.twiss" which lists the needed information for the sextupoles, skew sextupoles, octupoles in the ring.

use, period=rhic;

print;

select, flag=twiss, column=name,s, betx, bety, mux, muy, dx, k2l, k31, k2sl;

twiss, file="./test.twiss", save;

stop;

Then we run our script "IR_nonlinear_corr.py" to calculate the required correction strengths for each order of multipoles and for each interaction region. The correction strengths are given in MADX input format and is saved in the file "IR_nonlinear.result". It can be cut and pasted into the original MADX input file.

\section{Second order chromaticity correction}

To compensate the second order chromaticities, we sorted the 144 RHIC chromatic sextupoles into 8 subfamilies: SFPO, SFMO, SDPO, SDMO, SFPI, SFMI, SDPI, SDMI. There are several ways to correct the second order chromaticities with the 8 sextupole families [13]. In the tracking lattice model, we normally 
use MAD8 to find the correction strengths since it takes much less computing time than MADX. We have a converter script "madxTomad8.py" to generate the input files for MAD8 from the file "parameters_input". The command block in MAD8 to correct the second order chromaticities is

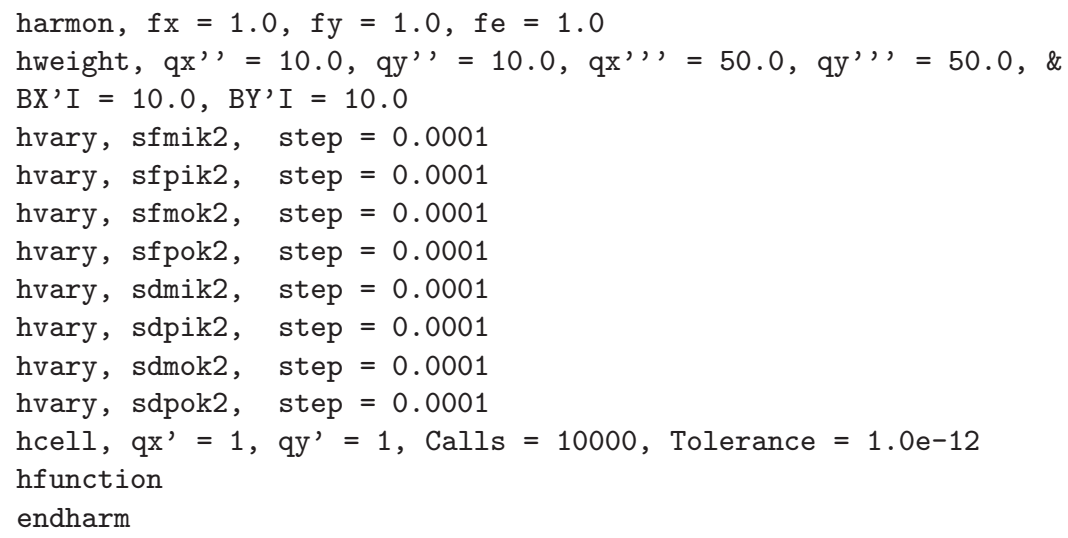

For the low $\beta^{*}$ lattice, the correction strengths from MAD8 sometime are overshot and the correction strengths for different sextupole families are not well balanced. To overcome them, we also use 4-knob method [14] to minimize the second order chromaticities.

The 4-knob method is valid for the second order chromaticity correction when the betatron phase advances of one arc FODO cell are around 90 degrees. The 4 knobs are the 4 pairs of chromatic sextupole families ( SFPO and SFMO ), (SFPI and SFMI) ( SDPO and SDMO ) and (SDPI and SDMI). In each correction step we adjust the two families of one pair with same amount absolute strength but with opposite signs. Normally the two pairs (SFPO and SFMO) and (SFPI and SFMI) have larger effects on the horizontal second order chromaticity, while the two pairs (SDPO and SDMO) and (SDPI and SDMI) have larger effects on the vertical second order chromaticities. The correction of second order chromaticities with the 4-knob method can be done manually or with a simple code written with SimTrack or Tracy-II. The correction strengths are typed or cut-pasted into the MADX input file.

\section{Third order resonance correction}

The current working point for the RHIC polarized proton run is constrained between $2 / 3$ and $7 / 10$. The beam-beam effects from proton-proton interactions at IP6 and IP 8 push the core particles toward the $2 / 3$ resonance line. In the previous RHIC beam experiment, we were trying to compensate its resonance driving term $h_{30000}$ with the local IR sextupole correctors in IR6 and IR8 [13]. This correction is not used in the previous RHIC operation since the effect of the correction to the beam lifetime is not verified. Note that these sextupole local correctors are also used in the above local IR multipole error correction.

The driving term for the horizontal third order resonance $3 Q_{x}$ is defined as

$$
h_{30000}=-\frac{1}{24} \sum_{i=1}^{N}\left(k_{2} d l\right)_{i} \beta_{x, i}^{3 / 2} e^{i 3 \mu_{x, i}} .
$$

The summation is done with all the sextupole components in the ring. It is calculated at the starting point of the lattice, that is, at IP6 for RHIC. We wrote a code with Tracy-II to correct the real and imaginary parts of $h_{30000}$ simultaneously with the 4 IR sextupole correctors in the IR6 and IR8 [15]. After that, the correction strengths are typed or cut-pasted into the MADX input file.

\section{Installing closed orbit deviations}

In the previous RHIC dynamic aperture calculation, the closed orbit is not included in the lattice model. To generate a realistic closed orbit, we require: 1) there is no clear betatron tune spectrum in the closed orbit along the ring, 2) the rms orbit should be closed to $0.3 \mathrm{~mm}$ which is what we have achieved in the routinely RHIC pp run operation.

Closed orbit deviations can be introduced by assigning random strengths to dipole correctors or by assigning random transverse position shifts to quadrupoles. The orbit correction to the deviated closed orbit 
can be done with SVD, best correctors and sliding bumps and so on [16]. After some tests, we decided to generate an initial closed orbit by assigning random strengths to the dipole correctors. Then we use sliding-bump method to bump down the local orbit which is larger than $0.5 \mathrm{~mm}$. After that, we scale the strengths of dipole correctors to obtain $0.3 \mathrm{~mm}$ orbit rms along the ring. With the sliding-bumps along the ring, we are able to remove the betatron oscillation spectrum in the closed orbit.

Figure 1 shows one example of the initial horizontal orbit, the corrected orbit with sliding bumps, and the final orbit for tracking which has $0.3 \mathrm{~mm} \mathrm{rms}$ orbit along the ring. From Figure 1, the initial horizontal orbit generated with random strengths to the horizontal dipole kickers displays a clear betatron oscillation in the orbit along the ring. After sliding bump correction, the betatron spectrum in the orbit is eliminated.

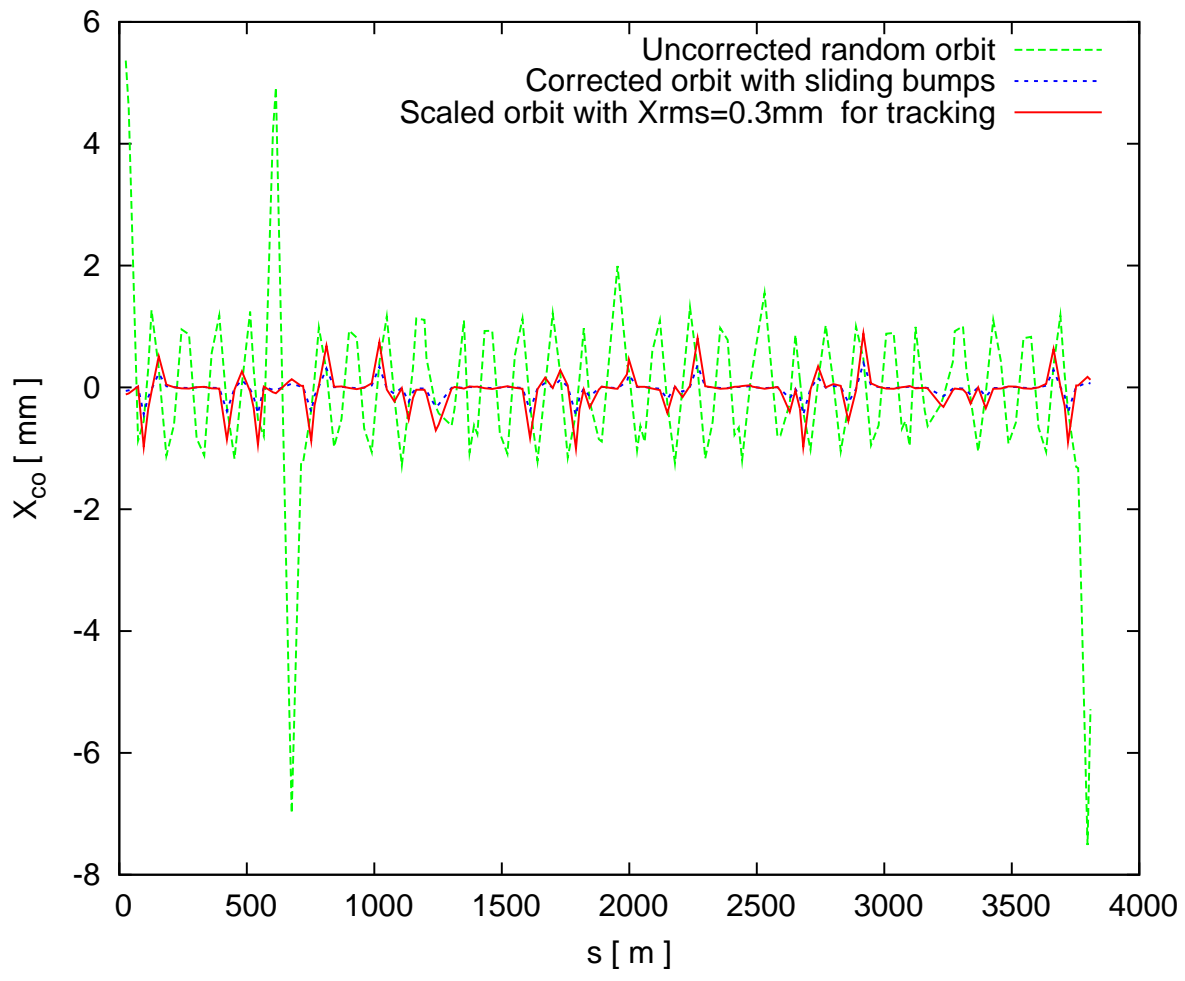

Figure 1: The artifical closed orbit generated for tarcking.

The above procedure is done in SimTrack since MADX doesn't have sliding bump method to correct orbit and in the MADX input file, all the dipole correctors are labeled as "KICKH" or "KICKV". In SimTrack, we first change the names of dipole kickers to their site_wide names before doing orbit correction. The resulting strengths of dipole correctors can be easily fed back to the file "parameters_input" if we will use SixTrack to do tracking.

Up to this step, all modifications to the base lattice model can be included in the input files of MADX. In the following we will modify the input files of SixTrack or program with SimTrack to include the tune ripples, physical aperture and 6-D beam-beam. Sixtrack input files can be generated in MADX as we mentioned above.

\section{Tune ripples}

Tune ripples were observed in RHIC operation [17]. Tab. 6 lists the tune ripples preliminarily measured with phase-locked loop (PLL) tune meter. To include them in the tracking lattice model, we insert an AC quadrupole at IP6 to generate the same amount of tune oscillation amplitude with a certain frequency. Since we use one $\mathrm{AC}$ quadrupole to generate the tune ripple, the tune oscillation will appear in both horizontal and vertical planes with similar amount since $\beta_{x}^{*}$ and $\beta_{y}^{*}$ are close at IP6.

Tune ripples can't be included in the input file of MADX since MADX doesn't have such type of element. If we use SixTrack to do tracking, we introduce power supply ripple to quadrupoles to generate tune ripple. 
Table 2: Tune ripples observed in the RHIC [17].

\begin{tabular}{lcc}
\hline \hline $\begin{array}{l}\text { Frequency } \\
{[\mathrm{Hz}]}\end{array}$ & $\begin{array}{c}\text { One period } \\
{[\text { Turns }]}\end{array}$ & $\begin{array}{c}\text { Amplitude } \Delta Q_{\max } \\
{\left[10^{-5}\right]}\end{array}$ \\
\hline 10 & 7819.62 & 25.0 \\
60 & 1303.27 & 2.0 \\
120 & 651.64 & 3.0 \\
360 & 217.12 & 2.0 \\
720 & 108.61 & 2.0 \\
\hline \hline
\end{tabular}

These AC quadrupoles' names have to be edited into fort.2 and their strength parameters in fort.3. In SimTrack, we directly insert an AC multipole at the IP6 to generate the tune ripples.

\section{Physical aperture}

In the RHIC opertaion, several one-plane and dual-plane collimators are routinely used in the IR8. For simplicity, especially in a long-term tracking, we only apply physical aperture check at IP6 each turn. In SixTrack and SimTrack, we can include a rectangle collimator at IP6. And in SimTrack, we can also check the particle's Courant-Snyder betatron actions to decide whether it is lost. Normally we set the aperture to $6 \sigma \mathrm{s}$, which is slightly larger than what was used in the operation.

\section{Beam-beam interaction}

In the previous RHIC dynamic aperture calculation we model the beam-beam interactions as a zero-length 4-D transverse kick. However, considering the RHIC proton bunch length is comparable to the $\beta^{*}$ at IP6 and IP8, we do need 6-D beam-beam treatment.

The 6-D beam-beam treatment has been implemented in the SixTrack [18] but it is not fully tested. We bench marked the 6-D beam-beam treatment in SimTrack against that in the BBSIM [19] and they agreed well. However, the 6-D beam-beam treatment in SimTrack currently doesn't include the crossing-angle scheme and coupling effect.

In the future RHIC dynamic aperture calculation and lifetime simulation study, we will only use 6-D beam-beam treatment. We plan to cross-check the treatments of 6 -D beam-beam in SixTrack, SimTrack, BBSIM and to include the crossing-angle collision scheme and coupling effect in the 6-D treatment.

\section{Betatron phase shift}

In the study of the head-on beam-beam compensation in the RHIC, we want to check the effect of the betatron phase between the proton-proton interaction point IP8 and the proton-electron interaction point which is close to IP10. The easiest way is to introduces two artificial phase advance shifters before and after the e-lens to maintain the overall tunes and Twiss parameters.

This phase shifter can be represented by a $6 \times 6$ matrix. In the SixTrack and SimTrack, both support the element type of $6 \times 6$ matrix. The only difference is that SixTrack uses $\left(x, x^{\prime}, y, y^{\prime}, z, \delta\right)$ coordinates while SimTrack uses $\left(x, p_{x}, y, p_{y}, z, \delta\right)$ coordinates. For SimTrack, the $6 \times 6$ matrix including dispersions is given in Ref. [20].

\section{References}

[1] Y. Luo, M. Bai, J. Beebe-Wang, R. Calaga, W. Fischer, C. Montag, S. Tepikian, D. Trbojevic, "Dynamic Aperture evaluation at the current working point for the RHIC polarized proton run", BNL C-AD AP Note 271, April, 2007.

[2] SixTrack code homepage, http://frs.home.cern.ch/frs/.

[3] R.D. Ruth, IEEE Trans. Nucl. Sci. vol. NS-30, pp.2669-2683 (1983). 
[4] MADX code homepage, http://mad.web.cern.ch/mad/.

[5] Steve Tepikian, private communication.

[6] A. Jain, D. Trbojevic, F. Dell, S. Peggs, P. Wanderer and J. Wei, BNL RHIC/AP/95, August, 1996.

[7] S. Trahern and F. Pilat, BNL RHIC/AP/99, June, 1996.

[8] R. Tomas, W. Fischer, A. Jain, Y. Luo and F. Pilat, in Proceedings of EPAC 2004, Lucerne, Switzerland.

[9] J. Beebe-Wang and A. Jain, in Proceedings of PAC 2007, Albuquerque, New Mexico, USA.

[10] M. Anerella, J. Cottingham, J. Cozzolino, P. Dahl, Y. Elisman, J. Escallier, H. Foelsche, G. Ganetis, M. Garber, A. Ghosh, C. Goodzeit, A. Greene, R. Gupta, M. Harrison, J. Herrera, A. Jain, S. Kahn, E. Kelly, E. Killian, M. Lindner, et al., "The RHIC magnet system", NIM A 499, pp 280-315, 2003

[11] F. Pilat, Y. Luo, N. Malitsky, V. Ptitsyn, "Beam-Based Non-Linear Optics Corrections", BNL C-AD AP Note 202, May 2005.

[12] J. Wei, Proceedings of the Workshop on LHC Interaction Region Correction Systems, BNL, May 6-7, 1999.

[13] Y. Luo, M. Bai, J. Beebe-Wang, J. Bengtsson, R. Calaga, W. Fischer, A. Jain, N. Malitsky, S. Peggs, F. Pilat, V. Ptitsyn, G. Robert-Demolaize, T. Satogata, S. Tepikian, D. Trbojevic, "Overview of magnetic nonlinear beam dynamcs in the RHIC", PAC09, Vancuver, Canada, May 2009.

[14] Y. Luo, S. Tepikian, W. Fischer, G. Robert-Demolaize, D. Trbojevic, "Sorting chromatic sextupoles for easily and effectively correcting second order chromaticity in the Relativistic Heavy Ion Collider", BNL C-AD AP Note 348, Jan. 2009.

[15] Y. Luo, J. Bengtsson, W. Fischer, and D. Trbojevic, "Simulation of proposed on-line third order resonance correction schemes", BNL C-AD AP Note 264, Jan 2007.

[16] Vadim Ptitsyn, private communications.

[17] P. Cameron, M. Minty, private communications.

[18] L.H.A. Leunissen, F. Schmidt. G. Ripken, Phys. Rev. ST Accel. \& Beams, v3, 124002(2000).

[19] T. Sen, Y-J.Kim, priviate communications.

[20] A. Chao, "Letcure Notes on Topics in Accelerator Physics", pp 282-283, March 2001. It is available at http://www.slace.stanford.edu/ achao/lecturenotes.html. 